\title{
Ultrashort Bradycardic Effect of Newly Synthesized Compounds
}

L. BARTOŠOVÁ, M. FRYDRYCH, G. VACULOVÁ, K. BERÁNKOVÁ, M. BÉBAROVÁ ${ }^{3}$, R. OPATŘILOVÁ ${ }^{1}$, V. STRNADOVÁ, P. MOKRÝ ${ }^{1}$, V. BRUNCLÍK ${ }^{2}$, J. KOLEVSKÁ ${ }^{2}$, J. KRČMÁR̆, L. BARTOŠIKOVÁ, T. FLORIAN, J. NEČAS

Department of Human Pharmacology and Toxicology, Department of Chemical Drugs ${ }^{1}$,

Pharmaceutical Faculty, Small Animal Clinic ${ }^{2}$, Faculty of Veterinary Medicine, University of Veterinary and Pharmaceutical Sciences, Brno, Czech Republic

Department of Physiology ${ }^{3}$, Faculty of Medicine, Masaryk University, Brno, Czech Republic

Received July 13, 2005

Accepted March 16, 2006

\begin{abstract}
Bartošová L., M. Frydrych, G. Vaculová, K. Beránková, M. Bébarováa , R.

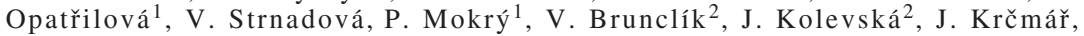
L. Bartošíková, T. Florian, J. Nečas: Ultrashort Bradycardic Effect of Newly Synthesized Compounds. Acta Vet. Brno 2006, 75: 183-196.
\end{abstract}

\begin{abstract}
Changes in the heart rate induced by four different doses of two newly synthesized potential ultrashort-action antagonists of beta adrenergic receptors were tested in 90 male laboratory Wistar rats. The isoprenaline-induced tachycardia model was used. Their effects were compared with those of esmolol. In the second part of the study, approximate electro-physiological measurements were made in vitro to assess the influence of the compounds tested on ion membrane currents in isolated ventricular cardiomyocytes. Both compounds demonstrated significant bradycardic effects in all concentrations tested compared with the control group, but they differed in the time of the onset of their action. Both newly synthesized compounds induced blockade of the fast sodium current $\left(\mathrm{I}_{\mathrm{Na}}\right)$ and potassium currents $\left(\mathrm{I}_{\mathrm{to}}, \mathrm{I}_{\mathrm{K} 1,} \mathrm{I}_{\mathrm{K}, \text { end }}\right)$.

Experimental pharmacology, rats, heart rate, ester-functional group, bradycardic effect, membrane currents, esmolol
\end{abstract}

Although attempts at using beta sympatolytic antagonists (beta blockers, beta adrenolytics, beta adrenergic receptors) have been made in many areas of medicine, they have gained a particularly important position in the treatment of cardiovascular diseases. They are indicated for the treatment of myocardial infarction, hypertension, arrhythmias and hyperthrophic cardiomyopathy (Frishman et al. 1984; McDevitt 1986; Hampton 1994). Betablockers, however, have also a number of side effects, e.g., bradycardia, hypotension, and they may have an aggravating effect on the heart failure, or rather, bronchospasm, peripheral vasoconstriction, fatigue, insomnia and depression. In order to avoid prolonged action, beta blocking agents with assumed ultra-short-acting period have been developed. Their pharmacological effects last only several minutes, and their administration as intravenous infusions in indicated patients in critical care situations is much safer than the use of longeracting beta-adrenergic blocking agents (Reilly et al. 1985; Gray 1988).

Ultra-short-acting beta blocking agents still form only a small fraction of a large group of beta-adrenolytics. At present, two ultra-short-acting beta blockers have been registered: esmolol (Brevibloc ${ }^{\circledR}$ inj.) (Gorczynski 1985) and landiolol (Onoact ${ }^{\circledR}$ inj.) (B arton et al. 1986). A third one, flestolol (Atarashi et al. 2000), is now being clinically tested. What they all have in common is a short elimination half-life achieved by the insertion of an ester group in the side chain on the aromatic core. This group is very rapidly hydrolyzed by plasma cholinesterases or by esterases present in the cytosole or erythrocyte membranes. With compounds of this type, gradual beta-blockade titration is possible and their clinical effects rapidly subside with the facultative termination of the therapy (Murthy et al. 1988). In the 
Czech Republic, the only registered drug with ultra-short-acting beta blocker esmolol is Brevibloc. Its active agent is esmolol hydrochloride. Ultra-short-acting beta blockers are used more and more frequently not only in emergencies where rapid control over the heart rate is necessary (sinus tachycardia, fibrillation and atrial flutter - Blanski et al. 1988) but also for the treatment and prophylaxis of tachycardia and hypertension caused by increased sympathetic activation under anaesthesia during surgery or in the postoperative stage (e.g. during laryngoscopy or intubation - Bensky et al. 2000).

Ultra-short-acting beta blockers are thus becoming an important therapeutic group, and new drugs exhibiting those characteristics are being developed.

On the basis of a pilot study (B artošová et al. 2003) and an analysis of the structure - the compound 44Bu may be assumed to have a beta-blocking rapid action. The aim of the present study was to find the relationship between the doses of compounds $44 \mathrm{Bu}$ and 444 and their pharmacological action. For the test of heart rate changes, the isoprenaline-induced tachycardia model was used. The effect on ion membrane currents was tested in the concentration of $10 \mu \mathrm{mol} \cdot \mathrm{l}^{-1}$ in vitro using isolated ventricular cardiomyocytes.

\section{Materials and Methods}

Tested Substances

Both tested compounds designated as $44 \mathrm{Bu}$ and 444 belong to newly synthesized compounds with a potential ultra-short beta- blocking action. Their ultrashort action is achieved by the incorporation of metabolically unstable ester functional group in the linking chain of the original aryloxyaminopropanol structure of the blocker (Mokrý et al. 2003). The tested substances are derivates of aryl-carbonyl-oxy-amino-propanols and their difference is in the different substituent in the aliphatic chain of the molecule - 44Bu has $n$-butyl and 444 terc-butyl (see Fig. 1).

Fig. 1. Chemical structure of the tested compounds<smiles>CCCCOC(=O)Nc1ccc(C(=O)OCC(O)C[NH+]([ClH+])CCCC)cc1</smiles>

Compound 44Bu

4 -Butoxycarbonylamino-benzoic acid 3 - butylamino - 2-hydroxy-propyl ester<smiles>COC(=O)CCc1ccc(OCC(O)CNC(C)C)cc1</smiles>

Esmolol

3-[4-(2 -Hydroxy-3-(isoproplylaminopropoxy)-phenyl]-propionic acid methyl ester<smiles>CCCCOC(=O)Nc1ccc(C(=O)OCC(O)C[NH+]([ClH+])C(C)(C)C)cc1</smiles>

Compound 444

4 -Butoxycarbonylamino-benzoic acid 3 - tert-butylamino - 2-hydroxy-propyl ester

The tested compounds were produced by a five-step synthesis as hydrochlorides. The structure was confirmed using ${ }^{1} \mathrm{H}-\mathrm{NMR}$ and ${ }^{13} \mathrm{C}-\mathrm{NMR}$ spectroscopy (Mokrý et al. 2001), (apparatus Varian Gemini), IR spectrophotometry (apparatus Nicolet Impact in $\mathrm{KBr}$ tablet). Purity was evaluated by TLC on silica gel (Kieselgel ${ }^{\circledR} 60 \mathrm{~F}_{254}$ Merck); mobile phase cyclohexane : ethanol : triethylamin $(4: 2: 1)$ $\mathrm{v} / \mathrm{v} / \mathrm{v}$ and by HPLC (column RP $\mathrm{C}_{18}$ Supelco ABZ+ ${ }^{\circledR}$ ); mobile phase methanol : water $(60: 40) \mathrm{v} / \mathrm{v}$. Basic physical indicators were assessed: melting point; solubility (see Table 1 and 2).

Both of the compounds are assumed to have a similar biotransformation as flestolol, which is also one of ultra- shortacting beta-blockers. The ester group in the molecule linking chain is hydrolyzed in plasma by plasmatic esterases (probably carboxylesterases), and two metabolites, 4-alkoxycarbonyl aminobenzoic acid and 3-alkylaminopropane-1,2-diol, are produced. 
Table 1. Basic characteristics of studied compounds

\begin{tabular}{|c|c|c|c|}
\hline Compound & Summary formula & $\mathrm{Mr}$ & Melting point ${ }^{\circ} \mathrm{C}$ \\
\hline Esmolol & $\mathrm{C}_{16} \mathrm{H}_{26} \mathrm{NO}_{4} \mathrm{Cl}$ & 331.83 & $85-86$ \\
\hline $44 \mathrm{Bu}$ & $\mathrm{C}_{19} \mathrm{H}_{31} \mathrm{~N}_{2} \mathrm{O}_{5} \mathrm{Cl}$ & 402.92 & $181.2-183.8$ \\
\hline 444 & $\mathrm{C}_{19} \mathrm{H}_{31} \mathrm{~N}_{2} \mathrm{O}_{5} \mathrm{Cl}$ & 402.92 & $187.9-189.9$ \\
\hline
\end{tabular}

Table 2. Solubility of the tested compounds

\begin{tabular}{|c|c|c|c|c|}
\hline Compound & purified water & ethanol & methanol & chloroform \\
\hline Esmolol & very easy & easy & very easy & - \\
\hline $44 \mathrm{Bu}$ & very low & easy & easy & very low \\
\hline 444 & very low & easy & very easy & very low \\
\hline
\end{tabular}

Solutions for intravenous administration of compounds $44 \mathrm{Bu}$ and 444 were made by dissolving the compounds in saline (sterile isotonic $0.9 \% \mathrm{NaCl}$ solution for infusion).

Experimental Animals

For in vivo tests, ninety Wistar strain rats with mean weight $246 \pm 25 \mathrm{~g}$ were used. They were conventional breed rats (Faculty of Medicine, MU Brno) and their housing complied with provisions of the Decree 286/1999 Coll. (temperature $20-24{ }^{\circ} \mathrm{C}$, humidity $40-60 \%, 12 \mathrm{~h}$ light $(200 \mathrm{~lx}$ max) and $12 \mathrm{~h}$ dark, housed in PVC cages with three rats per cage). The rats received standard feed $\mathrm{M}_{1}$ and water ad libitum. There were no restrictions regarding their access to feed and water before the experiment.

Methodology of the experiment was approved and monitored by the local Ethical Committee for Animal Welfare at the University of Veterinary and Pharmaceutical Sciences, under ref. No. 12609/2003-30/300 and 17739/200330/300.

\section{Experimental Design}

The experiment was designed as a comparative test of dose-dependent effects of compounds $44 \mathrm{Bu}$ and 444 on the heart rate. Their effects were compared with esmolol (Brevibloc ${ }^{\mathrm{R}}$ inj. Baxter Healthcare Ltd., UK). Compounds $44 \mathrm{Bu}$ and 444 and esmolol were tested in four doses at concentrations of $1.0 \mathrm{mg} \cdot \mathrm{kg}^{-1}\left(2.48 \mu \mathrm{mol} \cdot \mathrm{kg}^{-1}\right) ; 1.5 \mathrm{mg} \cdot \mathrm{kg}^{-1}$ (3.72 $\left.\mu \mathrm{mol} \cdot \mathrm{kg}^{-1}\right) ; 2.5 \mathrm{mg} \cdot \mathrm{kg}^{-1}\left(6,20 \mu \mathrm{mol} \cdot \mathrm{kg}^{-1}\right)$; and $3.5 \mathrm{mg} \cdot \mathrm{kg}^{-1}\left(8.69 \mu \mathrm{mol} \cdot \mathrm{kg}^{-1}\right)$ body weight of experimental animals. The tested compound was dissolved in $1 \mathrm{ml}$ saline. Each dose of the two newly synthesized compounds was tested on a group of eight rats, and each dose of esmolol was tested on a group of six rats. The control group consisted of six rats that were administered placebo ( $1 \mathrm{ml}$ saline).

\section{Experimental Procedure}

In vivo experiment: The rats were brought under general anaesthesia by the administration of a mixture of $1 \%$ ketamin solution (Narkamon ${ }^{\circledR}$ inj. Spofa) and 2\% xylazin solution (Rometar ${ }^{\circledR}$ inj. Spofa). The anaesthetic $\left(0.5 \mathrm{ml} .100 \mathrm{~g}^{-1}\right.$ b.w.) was administered intramuscularly to the femur region.

Heart rate changes were studied on the model of tachycardia induced by subcutaneous administration of $4 \mu \mathrm{g} \cdot \mathrm{kg}^{-1}$ (Czech formulary 2002) isoprenaline (Isuprel ${ }^{\circledR}$ inj. Abbott). The maximum heart rate averaging $152.42 \%$ of baseline was reached in 8 to $10 \mathrm{~min}$. Thus induced tachycardia remained stable for at least $30 \mathrm{~min}$ following the administration. The compounds tested were administered intravenously to the vena jugularis as a bolus dose of appropriate concentration and uniform volume $(1 \mathrm{ml})$. The compounds were administered over $20 \mathrm{sec}$.

The heart rate was monitored with electrocardiograph (SEIVA EKG Praktik) using the appropriate PC software. ECG measurements were made at predetermined intervals. The first measurement was made at the beginning of the experiment following the onset of total anaesthesia, the second $10 \mathrm{~min}$ after the subcutaneous administration of isoprenaline. That value corresponded to the initial level of tachycardia and was regarded as $100 \%$. The third measurement was made during the intravenous administration (designated 0). Further measurements were made at 30 -sec intervals in the first minute after administration, at 1 -min intervals from minute 1 to 6 , and at 2-min intervals between minute 6 and 20 following administration. The last measurement was made after $25 \mathrm{~min}$.

The heart rate values measured were then expressed as percentage of variation from the heart rate measured immediately before the administration of the tested compound regarded as $100 \%$.

In vitro experiment: Electrophysiological measurements of the effect of compounds $44 \mathrm{Bu}, 444$ and esmolol on ion membrane currents were made in ventricular cells enzymatically isolated from the hearts of Wistar laboratory rats. Currents were measured by the whole cell patch clamp technique at room temperature (Bahníková et al. 2002). Measurements were made at the compound concentration of $10 \mu \mathrm{mol} \cdot \mathrm{l}^{-1}$, which corresponds to $0.32 \mathrm{mg} \cdot \mathrm{kg}^{-1}$ body weight of the rats for compounds $44 \mathrm{Bu}$ and 444 and to $0.27 \mathrm{mg} \cdot \mathrm{kg}^{-1} \mathrm{~b} . \mathrm{w}$. for esmolol.

\section{Statistical Analysis}

Statistical computations were made in Microsoft Excel. For each value, the standard deviation was also computed. The non-paired Student's $t$-test was used for the comparisons between the effects of the control group 
and four different concentrations of compounds $44 \mathrm{Bu}, 444$ and esmolol. The test was used to determine the statistical significance of changes observed with the tested compounds in all four concentrations against both the control group and against heart rate changes induced by esmolol in the same concentration. The paired $t$-test was used to determine the statistical significance of heart rate changes against the initial tachycardia value for each group of results (groups with different doses).

\section{Results}

1. In vivo experiment - changes of the heart rate

Tests of the compound 44Bu

The time of the onset of action was similar in all three tested doses of $44 \mathrm{Bu}$, with the maximum between 2 and $3 \mathrm{~min}$ after i.v. administration. The least slowing effect 44Bu on the heart rate (by $4.91 \pm 3.35 \%$ compared with the baseline) was observed with the dose of $1.0 \mathrm{mg} \cdot \mathrm{kg}^{-1} \mathrm{~b}$.w. An increase in the concentration by $0.5 \mathrm{mg} \cdot \mathrm{kg}^{-1}$ almost tripled the effect of the compound (the heart rate slowed by $14.64 \pm 10.14 \%$ compared with the baseline). Further increase in concentration up to $2.5 \mathrm{mg} \cdot \mathrm{kg}^{-1}$ (a decrease by $14.06 \pm 8.43 \%$ compared with the baseline) did not, however, increase the effect of the substance significantly. In fact, at the concentration of $3.5 \mathrm{mg} \cdot \mathrm{kg}^{-1}$, the effect of $44 \mathrm{Bu}$ on the heart rate decreased markedly (the heart rate slowed down by only $8.06 \pm 6.87 \%$ compared with the baseline). The statistical significance of the heart rate response to different doses tested compared with placebo and with baseline values is given in Table 3. A graphical representation of the heart rate changes along the time line is shown in Fig. 2.

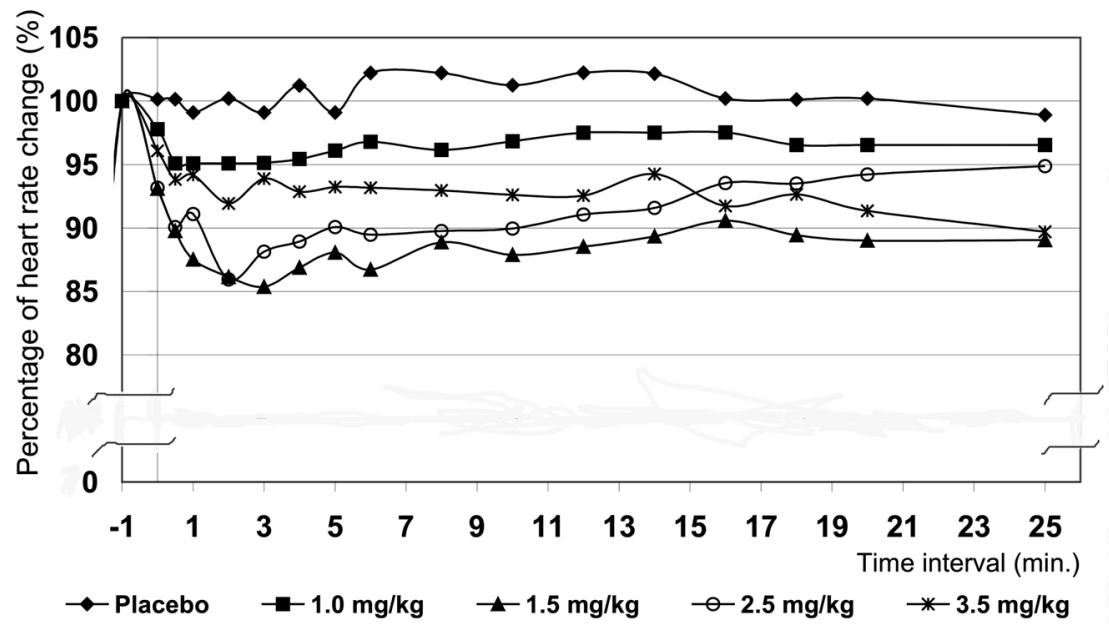

Fig. 2. Time line of heart rate changes (in \%) following administration of $44 \mathrm{Bu}$

Tests of the compound 444

The time of the onset of action was comparable in all three doses of the compound 444 tested, and the maximum effect was recorded between 1 and $2 \mathrm{~min}$ after administration. The heart rate slowing effects of the compound grew with increase in dosage until the concentration of $2.5 \mathrm{mg} \cdot \mathrm{kg}^{-1}$. The maximum slowing effects on heart rate were recorded after the administration of $1.0 \mathrm{mg} \cdot \mathrm{kg}^{-1}(7.74 \pm 1.33 \%), 1.5 \mathrm{mg} \cdot \mathrm{kg}^{-1}(12.27 \pm 7.66 \%)$ and 2.5 $\mathrm{mg} * \mathrm{~kg}^{-1}(17.34 \pm 5.07 \%)$ - see Fig. 3 . All the above values of the heart frequency decrease are related to the baseline. Administration of $3.5 \mathrm{mg} \cdot \mathrm{kg}^{-1}$ body weight of the compound 444 , however, caused major disturbances in the heart rhythm (AV blockades), ventricular 


\begin{tabular}{|c|c|c|c|c|c|c|c|c|c|c|c|c|c|c|c|c|c|c|c|c|c|}
\hline \multirow{12}{*}{ 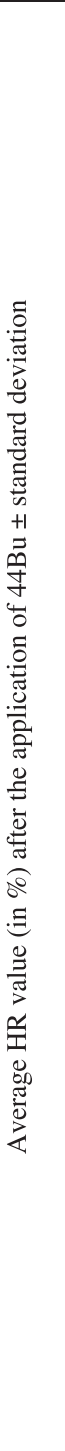 } & \multirow{3}{*}{ 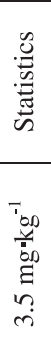 } & & + & $\stackrel{+}{+}$ & + & + & $=$ & $\begin{array}{l}+ \\
+\end{array}$ & $\mp$ & $\ddagger$ & + & 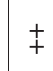 & $\mp$ & $\stackrel{+}{+}$ & $\stackrel{+}{+}+$ & $\begin{array}{l}+ \\
+ \\
+\end{array}$ & $\begin{array}{l}+ \\
+ \\
+\end{array}$ & $\stackrel{+}{+}$ & $\stackrel{+}{+}$ & \\
\hline & & \multicolumn{2}{|c|}{$\stackrel{\Xi}{\mathbf{z}}$} & $*$ & $*$ & $* *$ & $*$ & & & * & $*$ & $*$ & $* *$ & * & & & $*$ & $*$ & $*$ & & \\
\hline & & 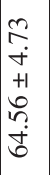 & 8 & $\begin{array}{l}0 \\
\infty \\
i \\
+1 \\
+1 \\
\infty \\
0 \\
0 \\
0\end{array}$ & $\begin{array}{l}m \\
\tilde{n} \\
+1 \\
+1 \\
\infty \\
\infty \\
\tilde{\sigma}\end{array}$ & $\begin{array}{l}a \\
\infty \\
i \\
+1 \\
\infty \\
\infty \\
\dot{J} \\
\dot{\alpha}\end{array}$ & 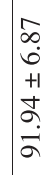 & $\begin{array}{l}0 \\
\text { ñ } \\
+ \\
\delta \\
\delta \\
\sigma\end{array}$ & & 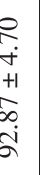 & $\begin{array}{l}\hat{\infty} \\
\dot{+} \\
+1 \\
+1 \\
\hat{\lambda} \\
\tilde{\alpha} \\
\alpha\end{array}$ & 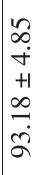 & 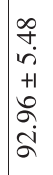 & $\begin{array}{l}= \\
\vec{n} \\
+1 \\
8 \\
\dot{0} \\
\dot{\alpha}\end{array}$ & 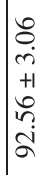 & $\begin{array}{l}\tilde{n} \\
\tilde{n} \\
+1 \\
\tilde{n} \\
\tilde{n} \\
\dot{\alpha}\end{array}$ & $\begin{array}{l}2 \\
\hat{+} \\
+1 \\
\stackrel{r}{a} \\
\dot{a}\end{array}$ & $\begin{array}{l}\dot{J} \\
\dot{+} \\
+1 \\
\dot{J} \\
\dot{\sigma} \\
\tilde{\sigma}\end{array}$ & 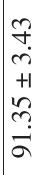 & 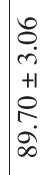 & \\
\hline &.$\ddot{g}$ & \multicolumn{2}{|c|}{$\stackrel{ \pm}{ \pm}$} & \pm & $+\underset{+}{+}$ & $\stackrel{+}{+}$ & $\stackrel{+}{+}$ & + & & 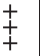 & $\begin{array}{l}+ \\
+ \\
+\end{array}$ & $\mp$ & + & \pm & + & $\neq$ & + & + & & & \\
\hline & 跑 & \multicolumn{2}{|c|}{$\stackrel{ \pm}{z}$} & $*$ & * & * & $\stackrel{*}{*} *$ & 苂 & & * & $\stackrel{*}{*}$ & * & $*$ & $*$ & $*$ & * & & & & & \\
\hline & 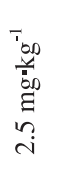 & \begin{tabular}{l}
$\infty$ \\
\hdashline \\
$=$ \\
+1 \\
+1 \\
$\infty$ \\
0 \\
0 \\
0
\end{tabular} & 8 & 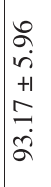 & $\begin{array}{l}\vec{\gamma} \\
0 \\
+1 \\
\infty \\
0 \\
\dot{8} \\
\delta\end{array}$ & $\begin{array}{l}+ \\
2 \\
i n \\
+1 \\
0 \\
\stackrel{.}{a} \\
\sigma\end{array}$ & $\begin{array}{l}m \\
f \\
\infty \\
+1 \\
+ \\
\sigma \\
\tilde{\infty}\end{array}$ & $\begin{array}{c}n \\
n \\
+ \\
+ \\
\infty \\
\infty\end{array}$ & & $\begin{array}{l}n \\
b \\
+1 \\
+1 \\
\vdots \\
\infty \\
\infty \\
\infty\end{array}$ & 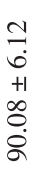 & \begin{tabular}{l}
$m$ \\
$\infty$ \\
$\infty$ \\
+1 \\
\multirow{f}{f}{} \\
$\infty$ \\
$\infty$
\end{tabular} & $\begin{array}{l}\infty \\
\infty \\
\alpha \\
+1 \\
\hat{\alpha} \\
\hat{\alpha} \\
\infty\end{array}$ & $\begin{array}{l}0 \\
\tilde{a} \\
\alpha \\
+1 \\
0 \\
2 \\
2 \\
\infty\end{array}$ & 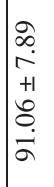 & $\begin{array}{l}\vec{\infty} \\
\alpha \\
+1 \\
0 \\
\tilde{\sigma} \\
\vec{\alpha}\end{array}$ & 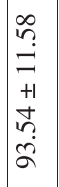 & 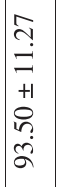 & 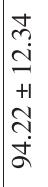 & $\begin{array}{l}\hat{a} \\
\dot{n} \\
+1 \\
+1 \\
\infty \\
\dot{d} \\
0\end{array}$ & \\
\hline & $\stackrel{\tilde{g}}{\tilde{v}}$ & \multicolumn{2}{|c|}{ \pm} & $\ddagger$ & $\underset{+}{+}$ & $\ddagger$ & + & + & & + & $\begin{array}{l}+ \\
\pm \\
+\end{array}$ & $\stackrel{+}{+}$ & $\stackrel{+}{+}+$ & 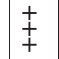 & $\stackrel{+}{+}$ & $\underset{\ddagger}{\ddagger}$ & $\begin{array}{l}+ \\
+ \\
+\end{array}$ & $\underset{f}{\ddagger}$ & $\underset{+}{+}$ & $\stackrel{+}{+}$ & \\
\hline & is & \multicolumn{2}{|c|}{$\stackrel{ \pm}{\beth}$} & $*$ & $*$ & $*$ & * & $*$ & & $*$ & * & * & $*$ & * & $*$ & F & $*$ & $*$ & $*$ & $*$ & \\
\hline & 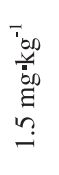 & 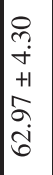 & $\underset{6}{8}$ & $\begin{array}{l}\vec{\sigma} \\
\dot{\sigma} \\
+1 \\
\stackrel{0}{a} \\
\alpha \\
\alpha\end{array}$ & $\begin{array}{l}\vec{\infty} \\
+ \\
+1 \\
+1 \\
\hat{N} \\
\infty \\
\infty\end{array}$ & 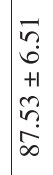 & $\begin{array}{l}2 \\
2 \\
\sigma \\
+1 \\
n \\
\\
\infty \\
\infty\end{array}$ & $\begin{array}{l}J \\
\\
0 \\
+ \\
0 \\
0 \\
2 \\
\infty\end{array}$ & & $\begin{array}{l}0 \\
+ \\
\vdots \\
+1 \\
+1 \\
\infty \\
\infty \\
\infty \\
\infty\end{array}$ & $\begin{array}{l}\infty \\
2 \\
1 n \\
+1 \\
1 \\
0 \\
\infty \\
\infty \\
\infty\end{array}$ & 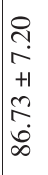 & $\begin{array}{l}\tilde{\Upsilon} \\
+ \\
+1 \\
\infty \\
\infty \\
\infty \\
\infty\end{array}$ & $\begin{array}{l}n \\
i n \\
+1 \\
+1 \\
2 \\
\infty \\
\infty\end{array}$ & $\begin{array}{l}\underset{N}{J} \\
\dot{+} \\
+1 \\
\tilde{N} \\
\tilde{\infty} \\
\infty\end{array}$ & 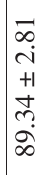 & $\begin{array}{l}\tilde{\sigma} \\
\dot{n} \\
+1 \\
\tilde{n} \\
\tilde{n} \\
\delta\end{array}$ & 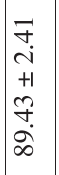 & $\begin{array}{l}0 \\
\stackrel{1}{a} \\
i \\
+1 \\
d \\
0 \\
\infty \\
\infty\end{array}$ & $\begin{array}{l}n \\
\sim \\
i \\
+1 \\
0 \\
0 \\
\infty \\
\infty\end{array}$ & \\
\hline &.$\tilde{n}$ & \multicolumn{2}{|c|}{$\stackrel{ \pm}{a}$} & + & $\ddagger$ & $\ddagger$ & + & + & & $\ddagger$ & $\ddagger$ & 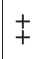 & $\ddagger$ & $\ddagger$ & + & + & & + & + & + & \\
\hline & 莺 & \multicolumn{2}{|c|}{ 艺 } & & * & & & & & & & & & & & & & & & & \\
\hline & 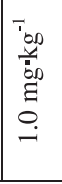 & 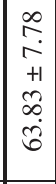 & $\begin{array}{l}0 \\
+1 \\
8 \\
8 \\
8 \\
0\end{array}$ & 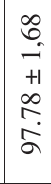 & $\begin{array}{l}n \\
m \\
m \\
+1 \\
\dot{\sigma} \\
\dot{\alpha} \\
\dot{a}\end{array}$ & $\begin{array}{l}\tilde{m} \\
\tilde{m} \\
+1 \\
o \\
\tilde{\sigma} \\
\tilde{a}\end{array}$ & $\begin{array}{l}n \\
m \\
m \\
+1 \\
o \\
o \\
\alpha\end{array}$ & 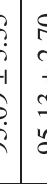 & 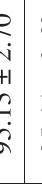 & $\begin{array}{l}\tilde{b} \\
\dot{j} \\
+1 \\
\dot{J} \\
\dot{v} \\
a\end{array}$ & $\begin{array}{l}= \\
\vec{i} \\
+1 \\
0 \\
0 \\
\dot{0} \\
\alpha\end{array}$ & $\begin{array}{l}\vec{b} \\
\stackrel{+}{+} \\
+1 \\
\infty \\
\alpha \\
\dot{\alpha}\end{array}$ & 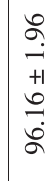 & $\begin{array}{l}8 \\
\dot{i} \\
+1 \\
+ \\
\infty \\
0 \\
\delta\end{array}$ & $\begin{array}{l}\text { in } \\
\stackrel{n}{+} \\
+1 \\
\stackrel{n}{n} \\
\stackrel{5}{a}\end{array}$ & 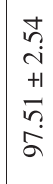 & $\begin{array}{l}\hat{n} \\
m \\
+1 \\
n \\
n \\
\hat{a}\end{array}$ & $\begin{array}{c}\vec{r} \\
i \\
+1 \\
n \\
n \\
2 \\
2\end{array}$ & $\begin{array}{l}\bar{r} \\
i \\
+1 \\
n \\
n \\
2 \\
\alpha\end{array}$ & $\begin{array}{l}\vec{i} \\
i \\
+1 \\
n \\
n \\
\tilde{o} \\
o\end{array}$ & \\
\hline 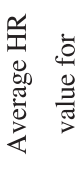 & 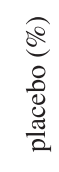 & $\begin{array}{l}\hat{m} \\
\tilde{n} \\
+1 \\
\hat{a} \\
g\end{array}$ & $\begin{array}{l}0 \\
+1 \\
8 \\
0\end{array}$ & 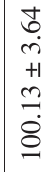 & $\begin{array}{l}n \\
\stackrel{n}{+} \\
+1 \\
m \\
\stackrel{8}{8}\end{array}$ & $\begin{array}{l}\tilde{n} \\
\tilde{+} \\
+1 \\
\stackrel{0}{0} \\
\stackrel{\alpha}{\sigma}\end{array}$ & $\begin{array}{l}\tilde{y} \\
0 \\
+1 \\
\tilde{1} \\
\tilde{8} \\
\delta\end{array}$ & 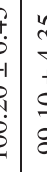 & 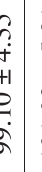 & $\begin{array}{l}\vec{\infty} \\
\dot{n} \\
+1 \\
\tilde{\imath} \\
\stackrel{0}{0}\end{array}$ & $\begin{array}{l}n \\
\tilde{+} \\
+ \\
+1 \\
\circ \\
\vdots \\
\sigma \\
\sigma\end{array}$ & 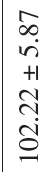 & 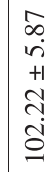 & $\begin{array}{l}8 \\
8 \\
0 \\
+1 \\
+ \\
+ \\
0 \\
0\end{array}$ & 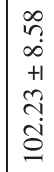 & $\begin{array}{l}2 \\
\stackrel{2}{\sim} \\
+1 \\
n \\
i \\
\tilde{\sigma} \\
\sigma\end{array}$ & 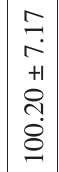 & \begin{tabular}{l}
$m$ \\
\hdashline \\
+1 \\
$\simeq$ \\
$\simeq$ \\
0 \\
0
\end{tabular} & 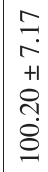 & $\begin{array}{l}0 \\
n \\
0 \\
+1 \\
8 \\
o \\
\infty \\
\alpha\end{array}$ & \\
\hline$H$ & & 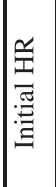 & 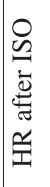 & 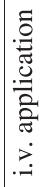 & 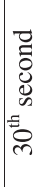 & 莺 & 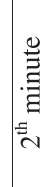 & 章 & & 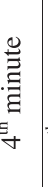 & 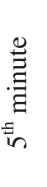 & 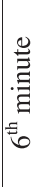 & 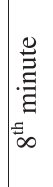 & 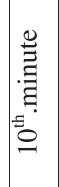 & 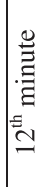 & 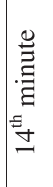 & 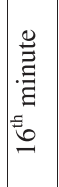 & 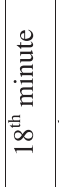 & 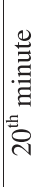 & 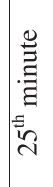 & II \\
\hline
\end{tabular}




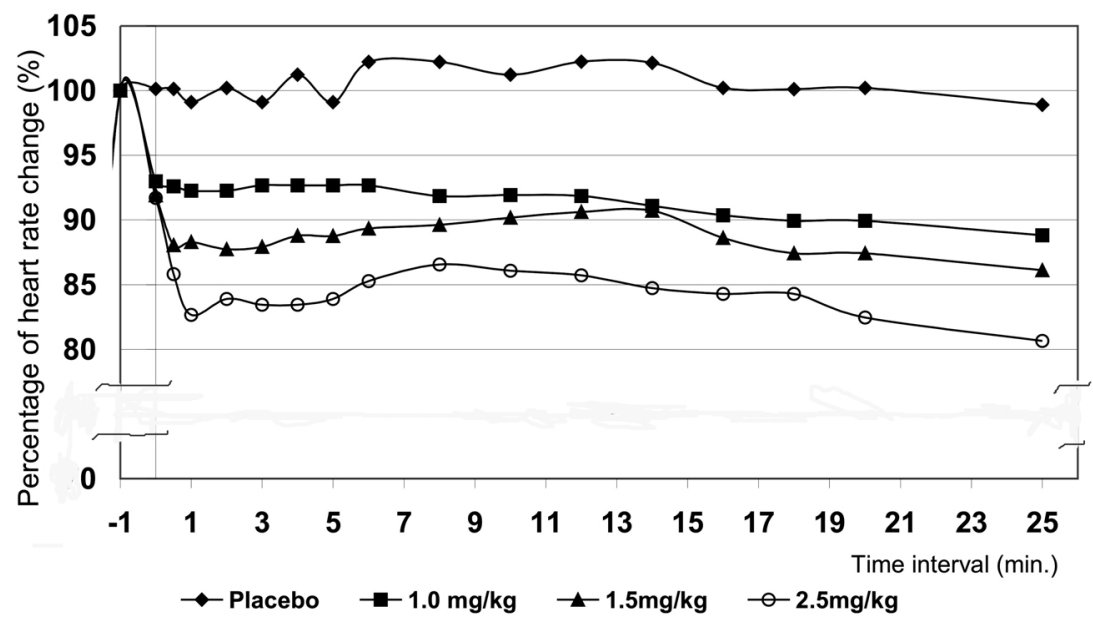

Fig. 3. Time line of heart rate changes (in \%) following administration of 444

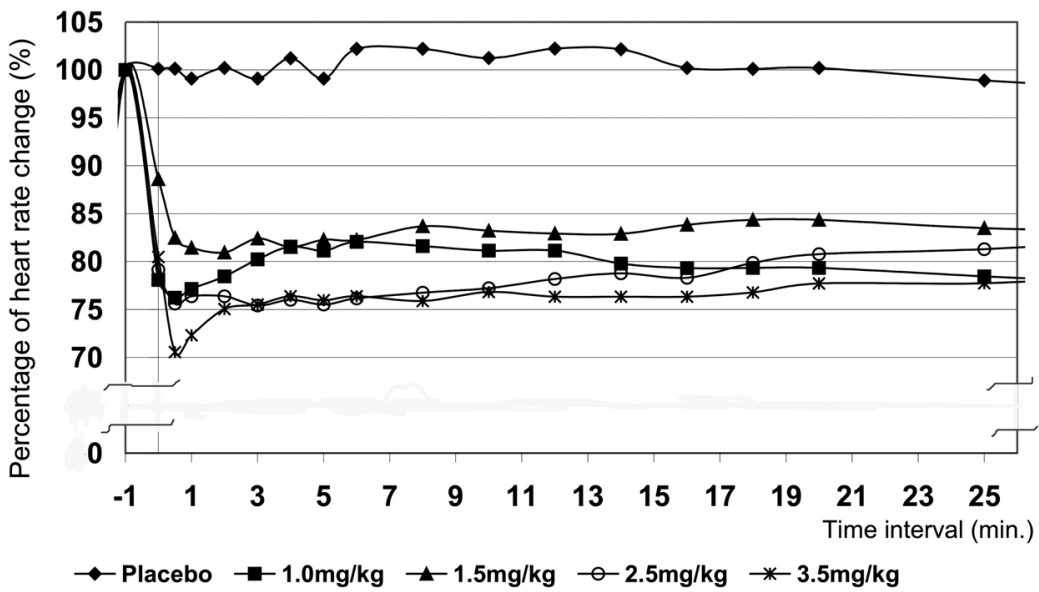

Fig.4. Time line of heart rate changes (in \%) following administration of esmolol

premature beats (bigeminies or triads) that caused the death of $50 \%$ of the animals. Because heart rate measurements in the rest of the rats were invalidated by sine rhythm lapses that rendered ECG records unusable, the results of that dose were not evaluated. Statistical significance of heart rate changes in response to individual doses tested compared with placebo and baseline values is given in Table 4.

Tests of esmolol

The time of the onset of action was comparable in all the doses tested. A statistically significant decrease in the heart rate compared with the control group was observed already during the i.v. administration of esmolol with a "low point" reached between 30 and 60 seconds after esmolol administration. Doses of $1.0 \mathrm{mg} \cdot \mathrm{kg}^{-1}, 1.5 \mathrm{mg} \cdot \mathrm{kg}^{-1}, 2.5 \mathrm{mg} \cdot \mathrm{kg}^{-1}$ and $3.5 \mathrm{mg} \cdot \mathrm{kg}^{-1}$ caused a decrease from the baseline of $23.77 \pm 6.64 \%, 19.06 \pm 7.24 \%, 24.58$ 


\begin{tabular}{|c|c|c|c|c|c|c|c|c|c|c|c|c|c|c|c|c|c|c|c|c|c|c|}
\hline \multirow{9}{*}{ 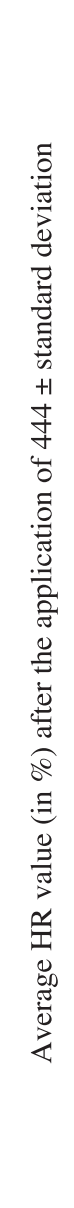 } & \multirow{3}{*}{ 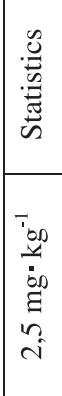 } & \multicolumn{2}{|c|}{$\stackrel{+}{ \pm}$} & \multirow[b]{2}{*}{ * } & + & $\stackrel{+}{+}$ & 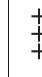 & & $\begin{array}{l}+ \\
+\end{array}$ & $\stackrel{+}{+}$ & $\stackrel{+}{+}$ & + & + & & $\stackrel{+}{+}$ & \pm & $\begin{array}{l}+ \\
+ \\
+\end{array}$ & $\stackrel{+}{+}$ & + & $\stackrel{+}{+}$ & + & $y$ \\
\hline & & \multicolumn{2}{|c|}{$\begin{array}{l} \pm \\
z\end{array}$} & & * & * & * & & $\begin{array}{l}* \\
* \\
*\end{array}$ & $\begin{array}{l}\text { 苂 } \\
*\end{array}$ & * & $\stackrel{*}{*} *$ & * & & $\stackrel{*}{*}$ & $\stackrel{*}{*}$ & * & * & $\begin{array}{l}\text { 苂 } \\
*\end{array}$ & $\stackrel{*}{*}$ & $\stackrel{*}{*}$ & 6 \\
\hline & & $\begin{array}{l}0 \\
i \\
i n \\
+1 \\
\dot{y} \\
\dot{\sigma} \\
\text { id }\end{array}$ & $\begin{array}{l}0 \\
+1 \\
8 \\
0\end{array}$ & 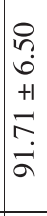 & $\begin{array}{l}\hat{0} \\
\dot{n} \\
+1 \\
\tilde{N} \\
\infty \\
\dot{\infty} \\
\infty\end{array}$ & $\begin{array}{l}5 \\
0 \\
i \\
+1 \\
+1 \\
0 \\
0 \\
\infty \\
\infty\end{array}$ & 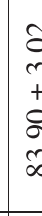 & & 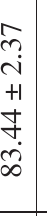 & $\begin{array}{l}\hat{n} \\
\text { i } \\
+1 \\
\dot{J} \\
\dot{\infty}\end{array}$ & $\begin{array}{l}m \\
\infty \\
+1 \\
+1 \\
\infty \\
\infty \\
\infty \\
\infty\end{array}$ & $\begin{array}{l}8 \\
\stackrel{8}{0} \\
i \\
+1 \\
\infty \\
\sim \\
\sim \\
\infty\end{array}$ & 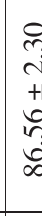 & & $\begin{array}{l}8 \\
\dot{0} \\
+1 \\
+1 \\
0 \\
\infty \\
\infty\end{array}$ & 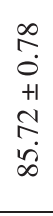 & $\begin{array}{l}\vec{b} \\
i \\
+1 \\
+1 \\
\stackrel{2}{+} \\
\dot{\infty}\end{array}$ & $\begin{array}{c}\hat{\infty} \\
i \\
+1 \\
+1 \\
\infty \\
\sim \\
\dot{\infty} \\
\infty\end{array}$ & 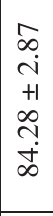 & $\begin{array}{l}\tilde{\sigma} \\
\tilde{m} \\
+1 \\
0 \\
+ \\
\dot{j} \\
\infty\end{array}$ & $\begin{array}{l}8 \\
n \\
n \\
+1 \\
0 \\
0 \\
\infty \\
\infty\end{array}$ & 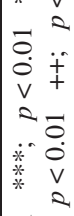 \\
\hline &. & \multicolumn{2}{|c|}{$\stackrel{ \pm}{ \pm}$} & $\stackrel{+}{+}$ & + & $\stackrel{+}{+}$ & $\ddagger$ & & + & $\stackrel{+}{+}$ & + & + & + & & + & + & + & $\mp$ & $\mp$ & + & + & \\
\hline & 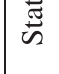 & \multicolumn{2}{|c|}{$\begin{array}{l} \pm \\
\mathrm{Z}\end{array}$} & * & * & * & * & & * & * & * & * & * & & * & * & * & * & $*$ & $*$ & $*$ & \\
\hline & 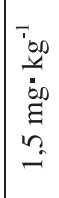 & $\begin{array}{l}0 \\
2 \\
n \\
+1 \\
+1 \\
\vdots \\
\dot{0} \\
\dot{0}\end{array}$ & $\begin{array}{l}0 \\
+1 \\
8 \\
0\end{array}$ & $\begin{array}{l}\hat{N} \\
\dot{m} \\
+1 \\
\sigma \\
\sigma \\
\sigma\end{array}$ & $\begin{array}{l}a \\
0 \\
+ \\
+1 \\
0 \\
0 \\
\infty \\
\infty\end{array}$ & $\begin{array}{l}0 \\
0 \\
0 \\
+1 \\
\text { ते } \\
\infty \\
\infty\end{array}$ & 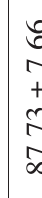 & & $\begin{array}{l}\infty \\
\infty \\
\infty \\
+1 \\
\alpha \\
\alpha \\
\infty \\
\infty\end{array}$ & $\begin{array}{l}m \\
\stackrel{n}{r} \\
+1 \\
\stackrel{2}{2} \\
\infty \\
\infty\end{array}$ & $\begin{array}{l}a \\
o \\
\infty \\
+1 \\
n \\
2 \\
\infty \\
\infty\end{array}$ & $\begin{array}{l}\hat{y} \\
\infty \\
+1 \\
\sim \\
\cdots \\
\infty \\
\infty\end{array}$ & $\begin{array}{l}d \\
0 \\
+ \\
+ \\
0 \\
\infty\end{array}$ & & $\begin{array}{l}\infty \\
\infty \\
\infty \\
+1 \\
\infty \\
0 \\
\dot{8}\end{array}$ & $\begin{array}{l}a \\
r \\
+1 \\
+1 \\
0 \\
\dot{8} \\
8\end{array}$ & $\begin{array}{l}\frac{a}{1} \\
+1 \\
\frac{1}{2} \\
\dot{8}\end{array}$ & $\begin{array}{c}\bar{n} \\
\infty \\
+1 \\
\overrightarrow{0} \\
\infty \\
\infty\end{array}$ & \begin{tabular}{l}
$n$ \\
$n$ \\
0 \\
+1 \\
\multirow{2}{*}{} \\
\multirow{2}{*}{} \\
$\infty$
\end{tabular} & \begin{tabular}{l}
$\infty$ \\
0 \\
0 \\
+1 \\
$\sim$ \\
\multirow{2}{*}{} \\
$\infty$ \\
$\infty$
\end{tabular} & $\begin{array}{c}0 \\
- \\
6 \\
+1 \\
\simeq \\
\simeq \\
0 \\
\infty\end{array}$ & \\
\hline & : & \multicolumn{2}{|c|}{ \pm} & $\stackrel{+}{+}$ & $\stackrel{+}{+}$ & + & + & & + & + & $\stackrel{+}{+}$ & $\stackrel{+}{+}$ & + & & + & $\stackrel{+}{+}$ & $\stackrel{+}{+}$ & $\stackrel{+}{+}$ & $\stackrel{+}{+}$ & $\stackrel{+}{+}$ & $\stackrel{+}{+}$ & \\
\hline & 矛 & \multicolumn{2}{|c|}{$\begin{array}{l} \pm \\
\text { Z }\end{array}$} & * & * & * & $*$ & & * & * & * & * & * & & $*$ & * & * & $\stackrel{*}{*}$ & $\stackrel{*}{*}$ & * & $*$ & \\
\hline & 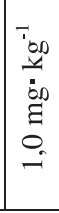 & 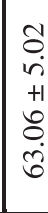 & $\begin{array}{l}0 \\
+1 \\
8 \\
0\end{array}$ & $\begin{array}{l}c \\
+ \\
\alpha \\
\sigma \\
\delta\end{array}$ & $\begin{array}{l}\text { సे } \\
+ \\
\frac{1}{0} \\
\delta\end{array}$ & 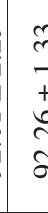 & 8 & 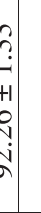 & $\begin{array}{l}n \\
0 \\
\\
+1 \\
\infty \\
0 \\
\text { a }\end{array}$ & 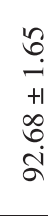 & $\begin{array}{l}n \\
0 \\
+ \\
\infty \\
\sigma \\
\sigma\end{array}$ & $\begin{array}{l}1 \\
5 \\
7 \\
5 \\
8 \\
8\end{array}$ & 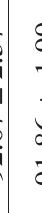 & 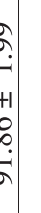 & 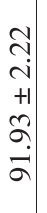 & 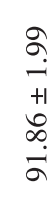 & $\begin{array}{l}\text { ç } \\
\text { i } \\
+1 \\
\stackrel{1}{0} \\
\text { aे }\end{array}$ & $\begin{array}{l}0 \\
\infty \\
i \\
+1 \\
0 \\
\approx \\
8\end{array}$ & 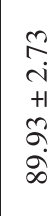 & 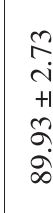 & $\begin{array}{l}n \\
n \\
n \\
+1 \\
\infty \\
\infty \\
\infty \\
\infty\end{array}$ & \\
\hline \multicolumn{2}{|c|}{ 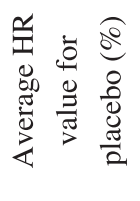 } & $\begin{array}{c}\hat{\sigma} \\
\text { nे } \\
+1 \\
+1 \\
\hat{\sigma} \\
\hat{\sigma}\end{array}$ & $\begin{array}{l}0 \\
+1 \\
8 \\
8\end{array}$ & $\begin{array}{l}\overrightarrow{0} \\
\dot{m} \\
+1 \\
m \\
\stackrel{1}{0}\end{array}$ & $\begin{array}{l}n \\
m \\
+ \\
+1 \\
m \\
0 \\
8 \\
8\end{array}$ & $\begin{array}{l}n \\
\text { r } \\
+1 \\
0 \\
\text { aे }\end{array}$ & $\stackrel{+}{\varrho}$ & & $\begin{array}{l}n \\
\tilde{+} \\
+ \\
+1 \\
0 \\
\vdots \\
\dot{\alpha}\end{array}$ & 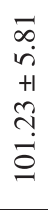 & $\begin{array}{l}n \\
\tilde{r} \\
+ \\
+1 \\
0 \\
\stackrel{0}{\sigma} \\
\sigma\end{array}$ & 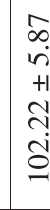 & $\begin{array}{l}n \\
1 \\
+ \\
2\end{array}$ & & $\begin{array}{l}8 \\
0 \\
0 \\
+1 \\
0 \\
+ \\
\dot{0} \\
0\end{array}$ & $\begin{array}{l}\infty \\
i n \\
\infty \\
+1 \\
\tilde{n} \\
i \\
0\end{array}$ & $\begin{array}{l}n \\
\stackrel{n}{r} \\
+1 \\
n \\
\text { a } \\
0\end{array}$ & 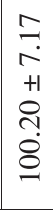 & 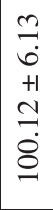 & 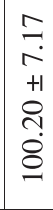 & $\begin{array}{l}0 \\
n \\
0 \\
+1 \\
8 \\
o \\
\infty \\
a\end{array}$ & 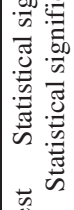 \\
\hline 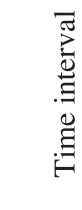 & & 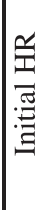 & 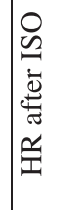 & 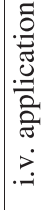 & 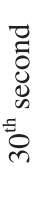 & 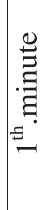 & 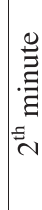 & ल & E⿱ & 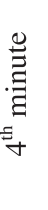 & 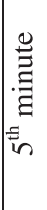 & 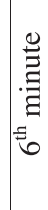 & 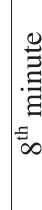 & 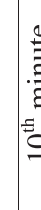 & है & 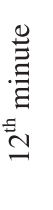 & 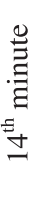 & 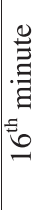 & 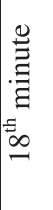 & 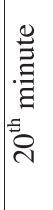 & 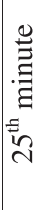 & II \\
\hline
\end{tabular}




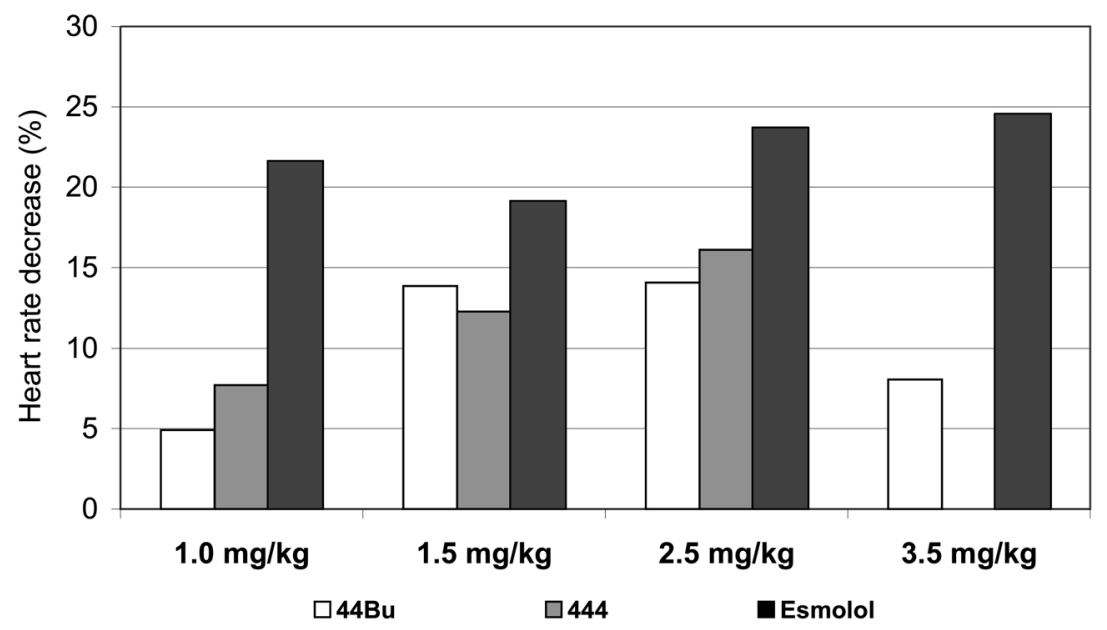

Fig. 5. Maximum slowing of heart rate (in \%) at $2 \mathrm{~min}$ following administration of the three compounds in all doses used

$\pm 9.36 \%$ and $29.45 \pm 3.28 \%$, respectively (see Fig. 4). This means that the bradycardic effect of esmolol was significantly greater than those of newly synthesized compounds at all doses tested (see Fig. 5). The only exception was with the dose at the concentration of $1.5 \mathrm{mg} \cdot \mathrm{kg}^{-1}$ where no significant difference in action between esmolol and the compound 444 was found. Statistical significance of heart rate changes in response to individual doses tested compared with placebo and baseline values is given in Table 5.

2. In vitro experiment - approximate measurements of ion membrane currents

Measurements of the following three currents were made: depolarization fast sodium current $\mathrm{I}_{\mathrm{Na}}$ and the main repolarization potassium currents: $\mathrm{I}_{\text {to }}$ (transient outward potassium current) $\mathrm{I}_{\mathrm{K} 1}$ (inward moiety of potassium current) and $\mathrm{I}_{\mathrm{K} \text {,end }}$ (potassium current measured at the end of $300 \mathrm{~ms}$ pulse). All three compounds were tested at the concentration of $10 \mu \mathrm{mol} \cdot \mathrm{l}^{-1}$. The time line of changes in ion membrane currents during experiments is shown in Figs 6 a-c and 7 a-c.

Measurements of fast sodium current $\mathrm{I}_{\mathrm{Na}}$

While $44 \mathrm{Bu}$ and 444 caused a $100 \%$ blockade of $\mathrm{I}_{\mathrm{Na}}$, esmolol caused only $45 \%$ blockade (see Figs $6 \mathrm{a}, 6 \mathrm{~b}$ and $6 \mathrm{c}$ ). The recovery of the current was gradual during the washout period in all three compounds.

Measurements of potassium repolarization currents

Transient outward potassium currents $\mathrm{I}_{\text {to }}$ were blocked more intensively by $44 \mathrm{Bu}(50 \%$ blockade) than by 444 (30\% blockade). On average, esmolol causes only $15 \%$ blockade of $\mathrm{I}_{\text {to }}$ (see Figs 7a, 7b a 7c). All blockades induced were fully reversible.

The most intensive blockade of the inward moiety of potassium current $\mathrm{I}_{\mathrm{K} 1}$ was caused by the compound 444 (36\% blockade), followed by esmolol (25\%) and 44Bu (15\%). Potassium current $\mathrm{I}_{\mathrm{K} \text {, end }}$ blockades by $44 \mathrm{Bu}, 444$ and esmolol were $30 \%, 20 \%$ and $15 \%$, respectively.

\section{Discussion}

Compounds designated as 44Bu and 444 are potential drugs with ultra-short beta-blocking action differing slightly in their molecule structures - 44Bu has $n$-butyl in its aliphatic chain, 444 has terc-butyl in it. In this project, we aspired to find out whether the above mentioned 


\begin{tabular}{|c|c|c|c|c|c|c|c|c|c|c|c|c|c|c|c|c|c|c|c|c|c|c|}
\hline \multirow{12}{*}{ 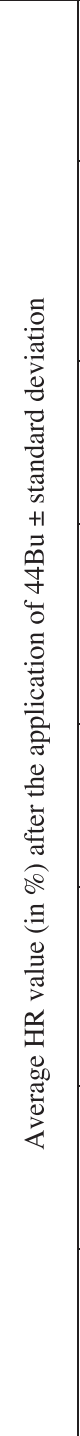 } & \multirow{3}{*}{ 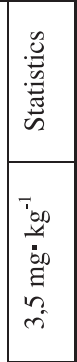 } & \multicolumn{2}{|c|}{$\stackrel{ \pm}{2}$} & $\stackrel{+}{+}$ & $\underset{+}{\ddagger}$ & 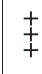 & + & + & & $\stackrel{+}{+}+$ & $\stackrel{+}{+}$ & + & 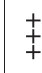 & + & & $\begin{array}{l}+ \\
+ \\
+\end{array}$ & $\stackrel{+}{+}$ & $\stackrel{+}{+}$ & $\stackrel{+}{+}+$ & $\ddagger$ & $\stackrel{+}{+}$ & \\
\hline & & \multicolumn{2}{|c|}{$\stackrel{ \pm}{z}$} & $\begin{array}{l}\text { 蒾 } \\
*\end{array}$ & 苂 & 苣 & * & * & & * & 苂 & 荬 & * & * & & * & * & $\stackrel{*}{*}$ & * & 莡 & $\stackrel{*}{*}$ & \\
\hline & & 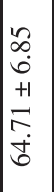 & $\begin{array}{l}0 \\
+1 \\
8 \\
0 \\
-1\end{array}$ & \begin{tabular}{l}
$\infty$ \\
2 \\
$n$ \\
+1 \\
+1 \\
\multirow{f}{f}{} \\
$\infty$ \\
$\infty$
\end{tabular} & 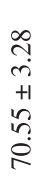 & $\begin{array}{c}0 \\
\infty \\
i \\
+1 \\
0 \\
\tilde{n} \\
\end{array}$ & 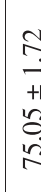 & $\begin{array}{l}\frac{3}{2} \\
+ \\
i n \\
i n\end{array}$ & & 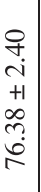 & 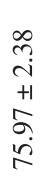 & $\begin{array}{l}\infty \\
\tilde{n} \\
+1 \\
\infty \\
\infty \\
\tilde{n} \\
\end{array}$ & $\begin{array}{l}\infty \\
\infty \\
i \\
+1 \\
\infty \\
\infty \\
i \\
i\end{array}$ & $\begin{array}{l}\delta \\
+ \\
+ \\
+\infty\end{array}$ & & 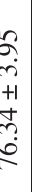 & 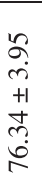 & 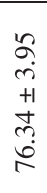 & $\begin{array}{l}\hat{\gamma} \\
\dot{\gamma} \\
+1 \\
\hat{\gamma} \\
\hat{\sigma}\end{array}$ & 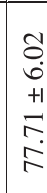 & 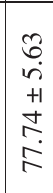 & \\
\hline &.$\stackrel{\mathscr{g}}{\mathscr{g}}$ & \multicolumn{2}{|c|}{$\stackrel{ \pm}{a}$} & + & $\stackrel{+}{+}$ & + & + & & + & + & + & + & + & + & & + & + & + & + & & + & $\dot{b}$ \\
\hline & 焉 & \multicolumn{2}{|c|}{$\stackrel{ \pm}{\mathrm{z}}$} & \begin{tabular}{|l|}
$*$ \\
$*$ \\
$*$
\end{tabular} & $\begin{array}{l}* \\
* \\
*\end{array}$ & 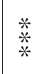 & * & * $*$ & & $\begin{array}{l}\text { * } \\
* \\
* \\
*\end{array}$ & $\begin{array}{l}\text { * } \\
* \\
*\end{array}$ & * & * * & *** & & * & $\begin{array}{l}\text { * } \\
* \\
*\end{array}$ & ** & 衬 & 苂 & * & 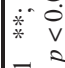 \\
\hline & 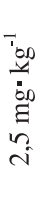 & 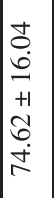 & $\begin{array}{l}0 \\
+1 \\
8 \\
0 \\
-1\end{array}$ & 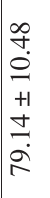 & $\begin{array}{l}\tilde{n} \\
\hat{\sigma} \\
+1 \\
\stackrel{1}{0} \\
\stackrel{2}{i}\end{array}$ & 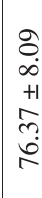 & $\begin{array}{l}8 \\
\infty \\
\infty \\
+1 \\
0 \\
0 \\
2\end{array}$ & $\begin{array}{l}5 \\
\text { co } \\
0 \\
+ \\
7 \\
5 \\
2\end{array}$ & 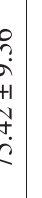 & \begin{tabular}{l}
$n$ \\
$\infty$ \\
$\sigma$ \\
+1 \\
8 \\
8 \\
0 \\
\hdashline
\end{tabular} & $\begin{array}{l}n \\
n \\
0 \\
+1 \\
n \\
n \\
n \\
n\end{array}$ & 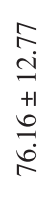 & 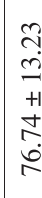 & $\begin{array}{l}\frac{1}{2} \\
\frac{1}{+} \\
\frac{1}{2}\end{array}$ & & 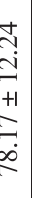 & $\begin{array}{l}\infty \\
\stackrel{\infty}{c} \\
+1 \\
\stackrel{1}{\circ} \\
\infty \\
\infty\end{array}$ & $\begin{array}{l}\vec{n} \\
m \\
n \\
+1 \\
\infty \\
\infty \\
\infty \\
\infty\end{array}$ & 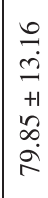 & $\begin{array}{l}\tilde{n} \\
\stackrel{+}{+} \\
+1 \\
\hat{f} \\
\dot{8}\end{array}$ & 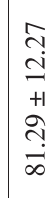 & $\begin{array}{l}\ddot{*} \\
\stackrel{*}{*} \\
\overline{8}\end{array}$ \\
\hline & $\tilde{u}$ & \multicolumn{2}{|c|}{$\stackrel{ \pm}{a}$} & + & + & + & + & + & $\begin{array}{l}+ \\
+\end{array}$ & + & + & + & $\stackrel{+}{+}$ & + & & + & $\stackrel{+}{+}$ & $\stackrel{+}{+}$ & $\stackrel{+}{+}$ & $\stackrel{+}{+}$ & $\stackrel{+}{+}$ & \\
\hline & 吾 & \multicolumn{2}{|c|}{$\stackrel{\Xi}{\text { z }}$} & 苂 & $\stackrel{*}{*}$ & $\stackrel{*}{*}$ & ** & * & & * & $\begin{array}{l}* \\
* \\
*\end{array}$ & 苂 & 苂 & * & & * & $\stackrel{*}{*}$ & $*$ & $* *$ & $\stackrel{*}{*}$ & $*$ & \\
\hline & 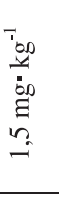 & $\mid \begin{array}{l}2 \\
\dot{m} \\
-1 \\
+1 \\
\infty \\
-0 \\
\dot{8} \\
0\end{array}$ & $\begin{array}{l}0 \\
+1 \\
8 \\
0\end{array}$ & $\begin{array}{l}\infty \\
0 \\
0 \\
+1 \\
0 \\
0 \\
\infty \\
\infty\end{array}$ & $\begin{array}{l}\text { q } \\
6 \\
+1 \\
+1 \\
\stackrel{+}{0} \\
\infty \\
\infty\end{array}$ & $\begin{array}{l}\hat{\jmath} \\
+ \\
+1 \\
\stackrel{2}{+} \\
\infty\end{array}$ & 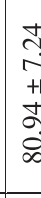 & $\begin{array}{l}\bar{z} \\
+ \\
7 \\
\delta\end{array}$ & 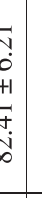 & 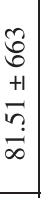 & 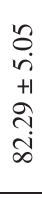 & 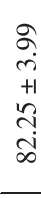 & 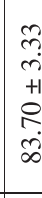 & $\begin{array}{l}\tilde{n} \\
+ \\
\tilde{n}\end{array}$ & & $\begin{array}{l}\Omega \\
\dot{n} \\
+1 \\
\vdots \\
\vdots \\
\infty \\
\infty\end{array}$ & 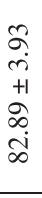 & \begin{tabular}{l}
\multirow{2}{\sigma}{} \\
$\dot{n}$ \\
+1 \\
$\hat{\infty}$ \\
$\infty$ \\
$\infty$ \\
$\infty$
\end{tabular} & $\begin{array}{l}n \\
f \\
+ \\
+1 \\
n \\
n \\
\tilde{\infty}\end{array}$ & $\begin{array}{l}n \\
f \\
f \\
+1 \\
n \\
m \\
\dot{\infty}\end{array}$ & $\begin{array}{l}\text { t } \\
0 \\
+1 \\
0 \\
0 \\
n \\
\infty \\
\infty\end{array}$ & : \\
\hline &. & \multicolumn{2}{|c|}{$\stackrel{ \pm}{a}$} & $\stackrel{+}{+}$ & + & $\begin{array}{l}+ \\
+\end{array}$ & + & $t$ & + & + & + & + & + & + & & + & + & $\stackrel{+}{+}$ & + & + & + & \\
\hline & 志 & \multicolumn{2}{|c|}{$\stackrel{ \pm}{z}$} & 苂 & $\stackrel{*}{*}$ & $\stackrel{*}{*} \underset{*}{*}$ & * & * & & $\stackrel{*}{*} *$ & $\stackrel{*}{*}$ & 苂 & * & * & & * & * & $\stackrel{*}{*} \underset{*}{*}$ & * & * & * & \\
\hline & 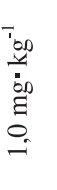 & $\begin{array}{l}\hat{n} \\
i \\
+1 \\
\dot{0} \\
\dot{0} \\
\dot{0} \\
i\end{array}$ & $\begin{array}{l}0 \\
+1 \\
8 \\
0\end{array}$ & 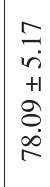 & 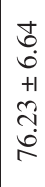 & 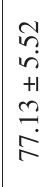 & $\begin{array}{l}0 \\
n \\
n \\
+ \\
+ \\
0 \\
+ \\
\infty\end{array}$ & 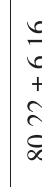 & & $\begin{array}{l}n \\
n \\
n \\
+1 \\
i \\
n \\
\infty\end{array}$ & $\begin{array}{l}\stackrel{+}{+} \\
+ \\
+1 \\
+ \\
+ \\
\infty \\
\infty\end{array}$ & 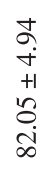 & $\begin{array}{l}\stackrel{J}{J} \\
+ \\
+1 \\
\stackrel{8}{8} \\
\stackrel{+}{\infty}\end{array}$ & $\begin{array}{l}0 \\
+ \\
+ \\
+ \\
+ \\
\infty\end{array}$ & & 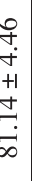 & 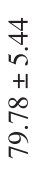 & $\begin{array}{l}n \\
n \\
n+1 \\
m \\
m \\
\tilde{n}\end{array}$ & 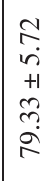 & $\begin{array}{l}\tilde{n} \\
\hat{b} \\
+1 \\
\tilde{m} \\
2\end{array}$ & $\mid \begin{array}{c}\overline{0} \\
+ \\
+1 \\
+ \\
\dot{0} \\
\infty\end{array}$ & 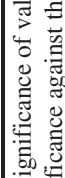 \\
\hline \multicolumn{2}{|c|}{ 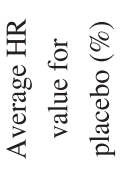 } & 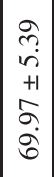 & $\begin{array}{l}0 \\
+1 \\
8 \\
0\end{array}$ & $\begin{array}{l}t \\
\dot{m} \\
+1 \\
+1 \\
n \\
0 \\
\dot{8}\end{array}$ & $\begin{array}{l}n \\
\tilde{r} \\
+1 \\
m \\
\ddot{8} \\
\dot{\delta}\end{array}$ & $\begin{array}{l}n \\
m \\
+ \\
+1 \\
0 \\
\stackrel{?}{a} \\
a\end{array}$ & 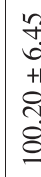 & \begin{tabular}{l}
$n$ \\
\multicolumn{1}{c}{+} \\
+ \\
+ \\
0 \\
8
\end{tabular} & & $\begin{array}{l}\vec{\infty} \\
\dot{n} \\
+1 \\
\tilde{n} \\
\dot{0} \\
-\end{array}$ & 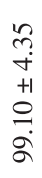 & 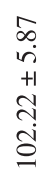 & 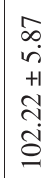 & 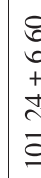 & & $\begin{array}{l}\infty \\
0 \\
\infty \\
+1 \\
\text { +1 } \\
\\
\vdots \\
0\end{array}$ & $\begin{array}{l}n \\
\stackrel{n}{2} \\
+1 \\
n \\
\stackrel{n}{0} \\
\delta\end{array}$ & 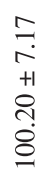 & $\begin{array}{l}2 \\
6 \\
+1 \\
1 \\
1 \\
8 \\
0\end{array}$ & 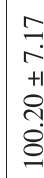 & $\begin{array}{l}0 \\
2 \\
b \\
+1 \\
8 \\
\infty \\
\infty \\
\alpha\end{array}$ & $\vec{\sigma}$ \\
\hline \multicolumn{2}{|l|}{ 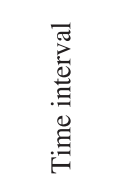 } & 舀 & 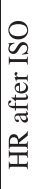 & 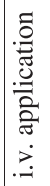 & 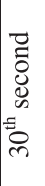 & 苞 & 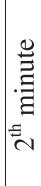 & 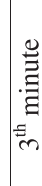 & 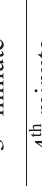 & 崖 & 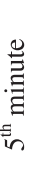 & 窇 & 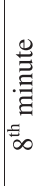 & 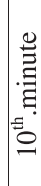 & 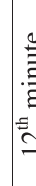 & 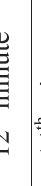 & 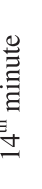 & 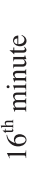 & 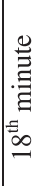 & 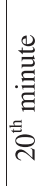 & 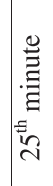 & $\mid \begin{array}{l}5 \\
\frac{5}{2} \\
\frac{11}{7}\end{array}$ \\
\hline
\end{tabular}




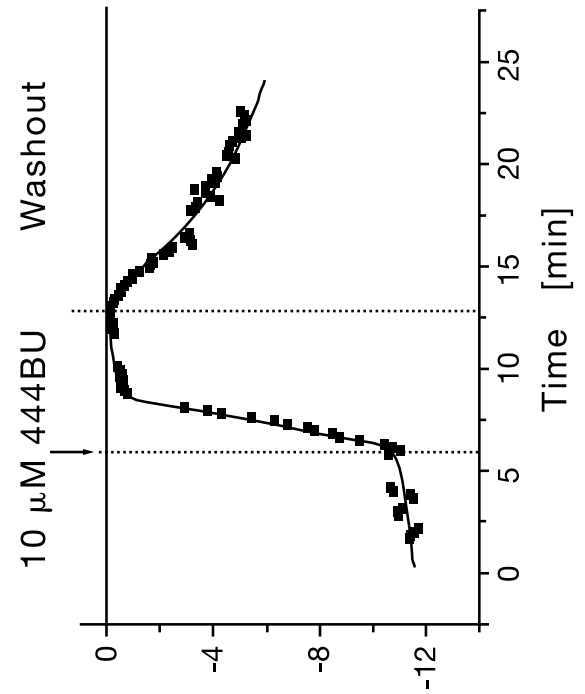

요 $[\forall \mathrm{U}]^{\mathrm{EN}}$
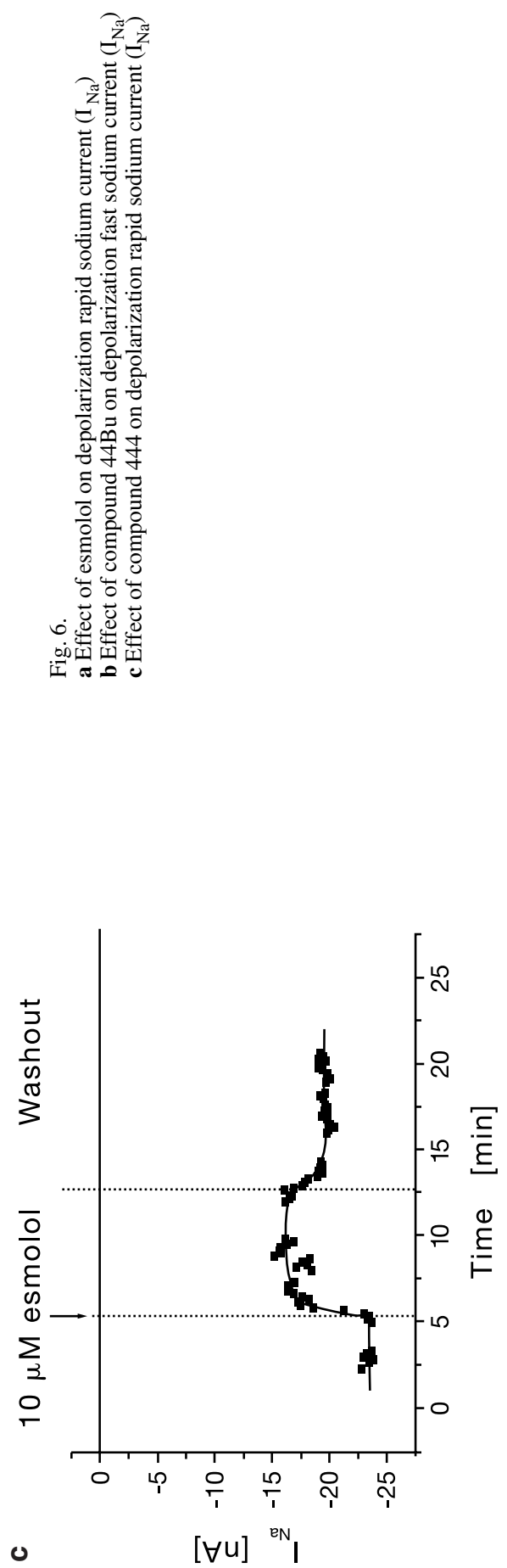
change in the chemical structure would affect the pharmacological effects of these compounds. Both compounds showed a very rapid onset of action at all doses tested. Statistically significant slowing of the heart rate compared with placebo was recorded already during i.v. administration, except for the lowest dose of $44 \mathrm{Bu}$ where a significant heart rate slowing was recorded only at 30 seconds. The results presented in this paper were, in part, published (Bartošová et al. 2004b).

Both $44 \mathrm{Bu}$ and 444 have thus bradycardic action with a rapid onset of their effect. Increasing doses of esmolol have an increasing effect on the heart rate. We observed the same direct relationship between the dose and the heart rate with the compound 444 until the dose at a concentration of $2.5 \mathrm{mg} \cdot \mathrm{kg}^{-1} \mathrm{~b}$.w. The administration of the compound 444 at the concentration of $3.5 \mathrm{mg} \cdot \mathrm{kg}^{-1}$, however, caused major heart rhythm disturbances characterized by AV blockade and ventricular extrasystoles, mainly bigeminies and trigeminies. Nevertheless, the lowest concentration of $1 \mathrm{mg} \cdot \mathrm{kg}^{-1}$ of $444 \mathrm{brought}$ about a significantly higher decrease of the heart rate than in $44 \mathrm{Bu}$, especially in the second half of the experiment. When a $2.5 \mathrm{mg} \cdot \mathrm{kg}^{-1}$ concentration was administered, bradycardic effect of the compound 444 lasted statistically significantly longer than that of $44 \mathrm{Bu}$.

Compound 44Bu caused a maximum decrease in the heart rate at the dose of $1.5 \mathrm{mg} \cdot \mathrm{kg}^{-1}$, and further dose increase failed to produce any significantly greater effect of the compound, i.e. further slowing of the heart rate. No significant differences between concentrations of $1.5 \mathrm{mg} \cdot \mathrm{kg}^{-1}$ and $2.5 \mathrm{mg} \cdot \mathrm{kg}^{-1}$ were found. This seems to indicate that the concentration of $1.5 \mathrm{mg} \cdot \mathrm{kg}^{-1} \mathrm{~b}$.w. was sufficient to produce bradycardic effects, and no further dose increase would produce a more intensive therapeutic effect. A comparison between the maximum heart rate slowing effects of the three compounds tested is shown in Fig. 5. Besides being bradycardic, the compound 44Bu tested in our study is also hypotensive. The administration of more that $3 \mathrm{mg} \cdot \mathrm{kg}^{-1}$ caused a rapid decrease in the systolic arterial blood pressure (Frydry ch et al. 2004), which then produced reflexive tachycardia. For that reason, the dose of $3.5 \mathrm{mg} \cdot \mathrm{kg}^{-1}$ had a lower bradycardic effect than expected. This phenomenon was not observed at lower concentrations (Frydrych et al. 2004) of 44Bu and any doses of the compound 444.

A comparison of our results with those reported in some previous experiments (B artošová et al. 2004a) showed no statistically significant differences in the bradycardic effect of $44 \mathrm{Bu}$ on the baseline heart rate and on the induced tachycardia. On the basis of these findings we may assume that the compound tested does not fully antagonize the effect of isoprenaline. In addition to beta-blocking activity, it probably has partial intrinsic sympathomimetic activity. It is, however, also possible that the heart rate slowing effect is due to its dromotropic effect on the myocardium, i.e. to a major prolongation of the PQ interval. Compared with esmolol, 44Bu causes an almost $39 \%$ greater prolongation of the PQ interval against the baseline, which is indicative of its greater slowing effect on the atrioventricular excitement conduction (Brunclík et al. 2003). The underlying cause of these electrophysiological changes is the ability of the newly synthesized compounds to completely block the depolarization fast sodium current $\left(\mathrm{I}_{\mathrm{Na}}\right)$, while esmolol is able to produce only about a $50 \%$ blockade. The newly synthesized compounds also have a more pronounced blocking effect on the repolarization outward potassium current (Ito). Membrane channels may be affected either directly by direct action on channels or indirectly by reduced activation of beta-adrenergic receptors. Both modes of action result in a decreased activation of membrane channels (Cheng et al. 1999; Ding et al. 2002; Lei et al. 2002; Bosch et al. 2002).

It thus seems that, however being incapable of topping the bradycardic effect of esmolol in vivo, both of the tested compounds display a much more significant blocking action on 


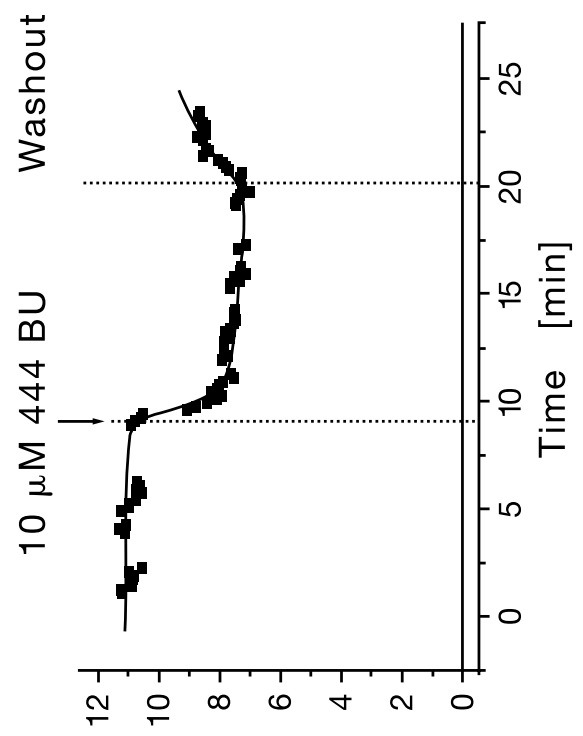

ค $[\forall u]^{\text {아 }}$
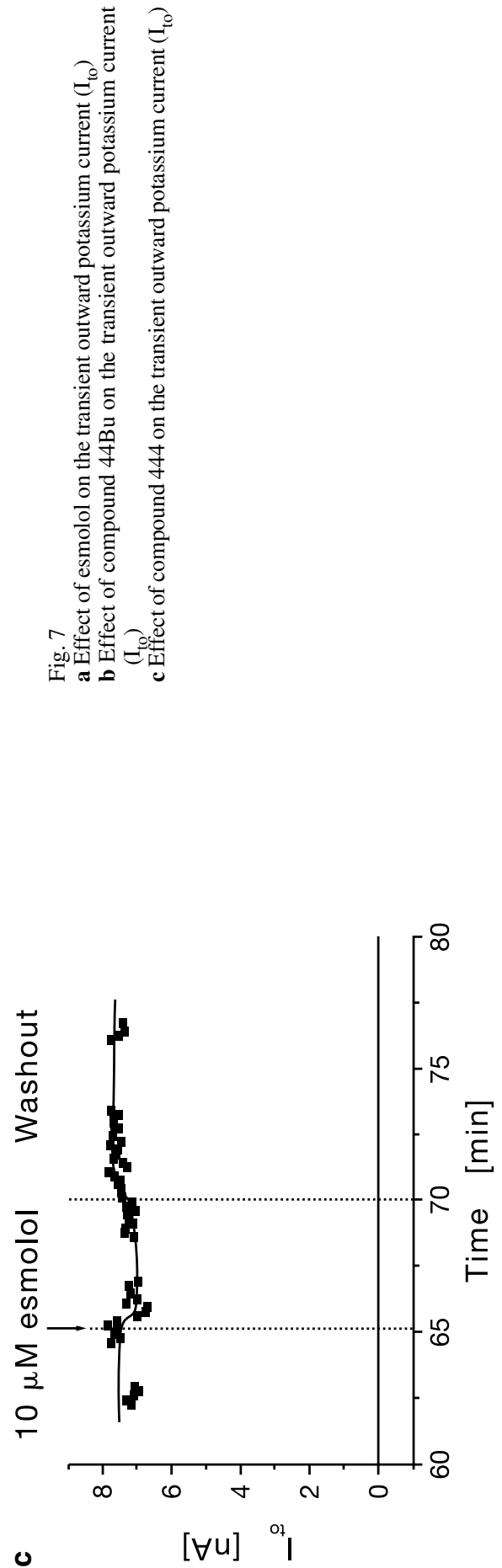
the ion membrane currents during in vitro experiments. That leads to the conclusion that they may have an important antiarrhythmic effect. We plan to explore that in further research.

\section{Ultrakrátký bradykardický účinek nově syntetizovaných sloučenin}

Na devadesáti normotenzních laboratorních potkanech kmene Wistar samčího pohlaví byly testovány ve čtyřech rozdílných dávkách změny srdeční frekvence dvou nově syntetizovaných potenciálních ultrakrátce působících antagonistů beta adrenergních receptorů. Změna srdeční frekvence byla testována na modelu isoprenalinem indukované tachykardie. Jejich efekt byl srovnáván s účinkem esmololu. Ve druhé části práce jsme provedli orientační elektrofyziologické měření vlivu testovaných látek na iontové membránové proudy izolovaných komorových kardiomyocytů in vitro. Obě látky vykazují ve všech testovaných koncentracích signifikantní bradykardický efekt vůči placebu, ale liší se v rychlosti nástupu účinku. Obě nově syntetizované látky, vyvolávají blokádu sodíkového depolarizačního proudu $\mathrm{I}_{\mathrm{Na}}$ a draslíkových proudů $\left(\mathrm{I}_{\mathrm{to}}, \mathrm{I}_{\mathrm{K} 1}, \mathrm{I}_{\mathrm{K} \text {,end }}\right)$.

\section{Acknowledgement}

This study was supported by grant 203/03/D182 and 305/06/0863 of the GA CR.

\section{References}

ATARASHI H, KURUMA A, YASHIMA M, SAITOH H, INO T, ENDOH Y, HAYAKAWA H 2000: Pharmacokinetics of landiolol hydrochloride, a new ultra-short acting beta-blocker, in patients with cardiac arrhythmias . Clin Pharmacol Ther 68: 143-50

BAHNÍKOVÁ M, MATEJOVIČ P, PÁSEK M, ŠIMURDOVÁ M, ŠIMURDA J 2002: Ajmaline-induced block of sodium current in rat ventricular myocytes. Scripta medica 75: 169-177

BARTON SD, BURGE J, TURLAPATY P, LADDU AR 1986: Flestolol: an ultra-short-acting beta-adrenergic blocking agent. J Clin Pharmacol 26: Suppl A, A36-A39

BARTOŠOVÁ L, FRYDRYCH M, MOKRÝ P, BRUNCLÍK V, KOTOLOVÁ H 2003: Changes in heart rate after application of newly developed ultrashort acting beta-adrenergic blockers. Pharmazie 58: $841-842$

BARTOŠOVÁ L, FRYDRYCH M, MOKRÝ P, BRUNCLÍK V, BAHNÍKOVÁ M 2004a: Testing the Bradycardic Effect of Newly Synthesized Potential Ultrashort Beta-blockers on the Laboratory Rat. Ces slov Farm 53:47-51

BARTOŠOVÁ L, FRYDRYCH M, HULÁKOVÁ G, BERÁNKOVÁ M, STRNADOVÁ V, MOKRÝ P, BRUNCLÍK V, KOLEVSKÁ J, BÉBAROVÁ M 2004b: Efficacy of Newly Synthesized, 44Bu UltrashortActing, Beta-Adrenergic Antagonist to Isoprenaline-Induced Tachycardia - Comparison With Esmolol. Acta Vet Brno 73: 171-179

BENSKY KP, DONAHUE-SPENCER L, HERTZ GE, ANDERSON MT, JAMES R 2000: The dose related effects of bolus esmolol on heart rate and blood pressure following laryngoscopy and intubation. AANA J 68: 437- 42

BLANSKI L, LUTZ J, LADDU A 1988: Esmolol, the first ultra- short-acting i.v. beta blocker for use in critically ill patiens. Heart Lung 17: 80-89

BOSCH RF, SCHNECK AC, KIEHN J, ZHANG W, HAMBROCK A, EIGENBERGER BW, RUB N, GOGEL J, MELIS C, SEIDEL L, KUHLKAMP V 2002: Beta 3-Adrenergic regulation of an ion channel in the heartinhibition of the slow delayed rectifier potassium current $\mathrm{I}(\mathrm{Ks})$ in guinea pig ventricular myocytes. Cardiovasc Res 56: 393-403

BRUNCLÍK V, BARTOŠOVÁ L, SEDLÁK P, KOLEVSKÁ J, FRYDRYCH M, NEČAS J, BARTOŠÍKOVÁ L 2003: Electrophysiological effect of Ultrashort Beta-blockers on the Myocardium of Laboratory Rat. Čs Fys 52: Suppl. A4

CHENG J, NIWA R, KAMIYA K, TOYAMA J, KODAMA I 1999: Carvedilol blocks the repolarizing K+ currents and the L-type Ca2+ current in rabbit ventricular myocytes. Eur J Pharmacol 376: 189-201

CZECH FORMULARY, 2002: part 1(Český lékopis, 1.díl) (ČL 02): Grada Publishing, Praha

DING WG, TOYODA F, MATSUURA H 2002 : Blocking action of chromanol 293B on the slow component of delayed rectifier $\mathrm{K}(+)$ current in guinea-pig sino-atrial node cells. Br J Pharmacol 137: 253-262

FRISHMAN WH, FURBERG CD, FRIEDEWALD WT 1984: Beta-adrenergic blockade for survivors of acute myocardial infarction. N Engl J Med 310: 830-837

FRYDRYCH M, BARTOŠOVÁ L, FLORIAN T, NEČAS J, BARTOŠÍKOVÁ L, KRČMÁŘ J, MOKRÝ P, BRUNCLÍK V 2004: Influence of New Ultrashort-Acting Beta-Adrenergic Blockers on Systolic Blood Pressure in Rats. Acta Vet Brno 73: 181-185

GORCZYNSKI RJ 1985: Basic pharmacology of esmolol. Am J Cardiol 56: 3F-13F

GRAY RJ 1988: Managing critically ill patients with esmolol. Chest 93: 398-403 
HAMPTON JR 1994: Choosing the right beta-blocker. A guide to selection. Drugs 48: 549

LEI M, COOPER PJ, CAMELLITI P, KOHL P 2002: Role of the 293b-sensitive, slowly activating delayed rectifier potassium current, $\mathrm{I}(\mathrm{Ks})$, in pacemaker activity of rabbit isolated sino-atrial node cells. Cardiovasc Res $\mathbf{5 3}$ : 68-79

McDEVITT DG 1986: Pharmacological characteristics of beta blockers and their role in clinical practice. J Cardiovasc Pharmacol 8: 5

MOKRÝ P, CSÖLLEI J, RAČANSKÁ E, TUMOVÁ I, ZEMANOVÁ-DURMISOVÁ M 2001: Synthesis and study of potential beta-adrenergic blockers with ultrashort action. $30^{\text {th }}$ conference Synthesis and analysis of drugs Brno

MOKRÝ P, ZEMANOVÁ M, CSÖLLEI J, RAČANSKÁ E, TUMOVÁ I 2003: Synthesis and pharmacological evaluation of novel potential ultrashort-acting beta-blockers. Pharmazie 58: 18-21

MURTHY VS, FRISHMAN WH 1988: Controlled beta-receptor blockade with esmolol and flestolol. Pharmacotherapy 8:168-182

REILLY CS, WOOD M, KOSHAKJI RP, WOOD AJ 1985:Ultra-short acting beta-blockade: a comparison with conventional beta- blockade. Clin Pharmacol Ther 38: 579-85 